\title{
Characterization of photonic structures using visible and infrared polarimetry
}

\author{
J. Ferré-Borrull ${ }^{1}$, E. Garcia-Caurel ${ }^{2}$, S. M. Olaizola ${ }^{3}$, Z. Kral ${ }^{1}$, J. Pallarès ${ }^{1}$ and L. F. Marsal ${ }^{1}$ \\ ${ }^{1}$ DEEEA, Universitat Rovira I Virgili, 43007, Tarragona, Spain \\ ${ }^{2}$ LPICM, CNRS, Ecole Polytechnique, 91128, Palaiseau, France \\ ${ }^{3}$ CEIT and Tecnun (University of Navarra), 20018, San Sebastián, Spain
}

\begin{abstract}
Photonic Crystals are materials with a spatial periodic variation of the refractive index on the wavelength scale. This confers these materials interesting photonic properties such as the existence of photonic bands and forbidden photon frequency ranges, the photonic band gaps. Among their applications it is worth mentioning the achievement of low-threshold lasers and high-Q resonant cavities. A particular case of the Photonic Crystals is well-known and widely studied since a long time: the periodic thin film coatings. The characterization of thin film coatings is a classical field of study with a very well established knowledge. However, characterization of $2 \mathrm{D}$ and $3 \mathrm{D}$ photonic crystals needs to be studied in detail as it poses new problems that have to be solved. In this sense, Polarimetry is a specially suited tool given their inherent anisotropy: photonic bands depend strongly on the propagation direction and on polarization. In this work we show how photonic crystal structures can be characterized using polarimetry equipment. We compare the numerical modeling of the interaction of the light polarization with the photonic crystal with the polarimetry measurements. With the S-Matrix formalism, the Mueller matrix of a Photonic Crystal for a given wavelength, angle of incidence and propagation direction can be obtained. We will show that useful information from polarimetry (and also from spectrometry) can be obtained when multivariate spectra are considered. We will also compare the simulation results with Polarimetry measurements on different kinds of samples: macroporous silicon photonic crystals in the near-IR range and Laser-Interference-Lithography nanostructured photoresist.
\end{abstract}

This is an Open Access article distributed under the terms of the Creative Commons Attribution-Noncommercial License 3.0, which permits unrestricted use, distribution, and reproduction in any noncommercial medium, provided the original work is properly cited. 